\title{
Dispatching Rules with Cycle Time Prediction for Semiconductor Manufactury
}

\author{
Yin-Dong Wei, Qi-Di Wu, Li Li \\ College of Electronics and Information Engineering, \\ Tongji University, \\ Shanghai, China \\ E-mail: 1433204@tongji.edu.cn, lili@tongji.edu.cn
}

\begin{abstract}
Semiconductor manufacturing system is considered as one of the most complex manufacturing systems, due to its characteristics like multiple-entry and large-scale. As for scheduling a job, its remaining cycle time is an important factor to the decision, but current researches seldom study the influence of a job's remaining cycle time on the performance of the system. Therefore, in this paper a novel scheduling strategy with remaining cycle time prediction is proposed. The method has two steps. First, we use the product line data to establish the remaining cycle time prediction model. Second, when scheduling the job in bottleneck equipment, we use the model to predict the remaining cycle time for scheduling decision. Several online simulations show that the new strategy has improved by $8 \%$ in On-time delivery rate (abbreviated as MOV). Meanwhile, the performance of movement of the jobs (abbreviated as MOV) in one day is almost the same as the rule without remaining cycle time prediction.
\end{abstract}

Keywords-remaining cycle time prediction;semiconductor manufacturing system; scheduling

\section{INTRODUCTION}

Semiconductor manufacturing system is one of the most complex manufacturing systems and many researchers are focusing on its scheduling. The scheduling theory can be divided into two dimensions as a whole: the scheduling methods and the object which scheduling methods concerns. On one hand, various scheduling methods are used by researchers. The existing scheduling methods can be roughly grouped into the following categories: methods based on computational intelligence, operational research, discrete event simulation, heuristic rules and other methods. On the other hand, different researchers focus on different objects in the system. For example, some simply focus on the scheduling of the key equipment or regions, while others focus on the scheduling of the whole product line.

The most concerned scheduling objects are batch processing machines and bottleneck machines. For example, Chang et al. [1] applied a method which combined variable selection and LR three-stage of proportion method to schedule wet etching region with furnace equipment and provide the solutions on forming batches and determining the number of wafers in batches. Deng et al. [2] proposed some dispatching rules to bottleneck equipment scheduling which used adaptive fuzzy neural network to predict waiting time and the load in the system. As for the whole product line scheduling, Shi et al. [3] adopted dynamic dispatching scheme based on pheromone to optimize performance indicators, such as cycle time and movement of the jobs (abbreviated as MOV). They first adjusted the program parameters to make performance changes, and then picked up the rules which lead to excellent performance.

Many researchers also pay attention to dynamic dispatching rules. Among them, we found the remaining cycle time is an important factor to obtain an excellent rule for scheduling the system. But as to how to get the cycle time, various methods differ among researchers. For example, Chang et al. [4] applied a Self-Organizing Map (SOM) method to categorize real time situations and then used a Case-Based Reasoning (CBR) model to predict cycle time. At the same time, they used genetic algorithms to fine-tune the feature weights of the CBR model. Chen et al. [5] proposed a fuzzy-neural ensemble method to estimate the remaining cycle time and a geometric rule fusion approach to fuse two dispatching rules. Meida et al. [6] used maximized condition information to identify the factors which influence the cycle time. And then they used a selective native Bayesian classifier (SNBC) to select 20-dimensional independent factors to predict cycle time. The accuracy is close to other machine leaning methods, but this method is easier than other methods.

From the researches above, we can find that although the importance of predicting the remaining cycle time is emphasized, they seldom were used to directly affect the scheduling decisions.

In this article, firstly, we propose a simple dynamic dispatching rule to schedule the production line. In order to get enough data to model the remaining cycle time prediction model, a simulation semiconductor fabrication factory is established. Secondly, we use random forests to predict the remaining cycle time of a job. Thirdly, we incorporate the remaining cycle time prediction with the dynamic rules which we previously proposed. Finally, we compare the system performance with different rules, and draw the conclusions.

\section{DYNAMIC DISPATCHING RULES}

First, we propose a simple dynamic dispatching rule without considering remaining cycle time. The process is shown in figure 1. 


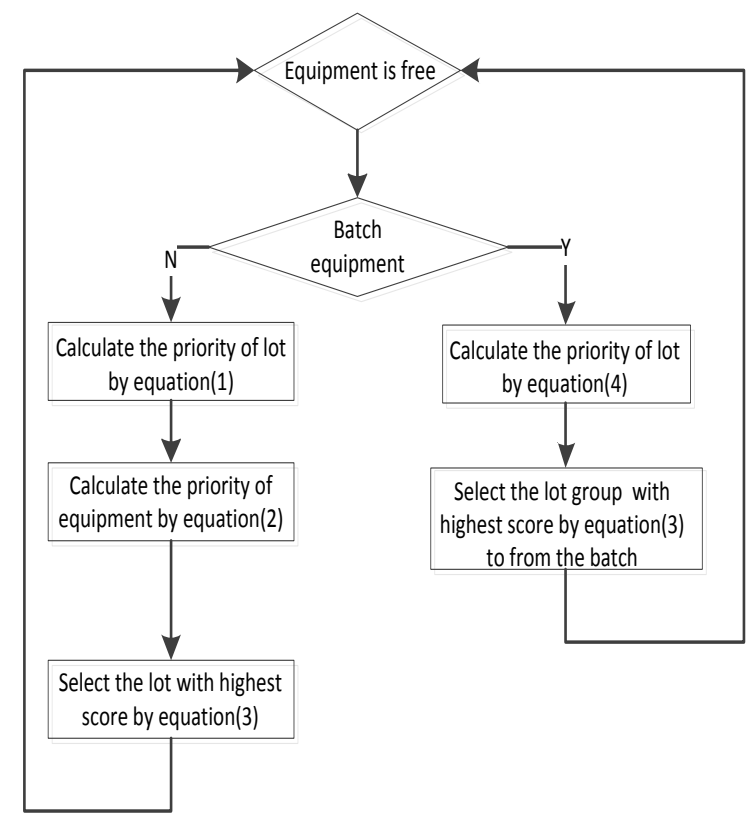

Figure1. Dynamic dispatching rule without remaining cycle time prediction.

Equations are as follows:

$$
\tau_{i}^{\mathrm{n}}(t)=\left\{\begin{array}{cc}
M A X & R_{i}^{n} * F_{n} \geq D_{n}-t \\
\frac{R_{i}^{n} * F_{n}}{D_{n}-t+1}-\frac{P_{i}^{n}}{\sum_{n} P_{i}^{n}} & R_{i}^{n} * F_{n} \geq D_{n}-\mathrm{t}
\end{array}\right.
$$

The meaning of equation (1) is as follows. $R_{i}^{n}$ is the remaining propose time, and $F_{n}$ is a constant. $D_{n}$ is the deadline of a job, and $\mathrm{t}$ is the current time. $P_{i}^{n}$ is the process time of job i processed at equipment n. So the first part is the ratio of theoretical remaining processing time and real remaining time of job $n$, which means that a larger ratio is more urgent. The second part means a job with short occupation time will be processed as soon as possible.

$$
\tau_{i d}^{n}(t)=\frac{P_{i d}^{n}}{T_{i d}}
$$

The meaning of equation (2) is as follows. $P_{i d}^{n}$ is the process time of job $\mathrm{n}$ at equipment id. $T_{i d}$ is the time when next equipment can be used. So the value of equation (2) represents the workload degree. If the value is greater than 1 , the equipment becomes a temporary bottleneck.

$$
S_{n}=\left\{\begin{array}{cc}
Q_{i}^{n} & t_{i}^{n}(t)=M A X \\
\alpha_{1} t_{i}^{n}(t)-\beta_{1} t_{i d}^{n}(t) & t_{i}^{n}(t) \neq M A X
\end{array}\right.
$$

The meaning of equation (3) is as follows. $Q_{i}^{n}$ is the time job $n$ processed at equipment $i$. That means when we schedule a job, we consider the due date of jobs, their occupation time on the machine and bottleneck level of the related downstream machines simultaneously to guarantee jobs' fast movement and on-time delivery performance.

$$
\tau_{i}^{n}(t)=\tau_{i}+\frac{t-l_{i}}{\text { canwaitime }_{i}}
$$

The meaning of equation (4) is as follows. $l_{i}$ is the time that job $\mathrm{i}$ put in the product line. canwaitime $_{i}$ means the time a job is to wait when forming the batch. $\tau_{i}$ is the priority of job i. That means if a job in the product line which is put at an early time and can't wait long will be processed as soon as possible.

\section{DATA COLLECTION AND CYCLE TIME PREDICTION}

\section{A. Data Collection}

First, we construct the manufacturing model to produce enough data at different conditions. The manufacturing model is the model of a real semiconductor manufacturing factory in Shanghai with nine manufacturing districts. In the simulations this paper chooses nine products with different cycle time which ranges from 22 days to two mouths when WIP is about 6500 at average to be produced. The dispatching rule is chosen FIFO. At the data collection process, this paper collects WIP, the number of hot jobs, the number of bottleneck equipment, the number of jobs waiting to be processed and the whole process time of these job at a certain district. So there are 44 variables we can choose to predict the remaining cycle time. The variables we choose are as follows. Table I shows the variables of the whole product line, and table II shows the variables in injection district(IM). In the other eight districts this paper chooses the same variables, so here we don't show them.

TABLE I. THE PRODUCT LINE VARIABLE

\begin{tabular}{|c|c|c|}
\hline No. & Variable name & The meaning of variable \\
\hline 1 & isHot & Whether the job of hot \\
\hline 2 & remainStep & $\begin{array}{c}\text { The number of steps remaining to } \\
\text { be processed }\end{array}$ \\
\hline 3 & prodid & The name of product \\
\hline 4 & wip & The number of job in product line \\
\hline
\end{tabular}

TABLE II. THE VARIABLE IN IM DISTRICT

\begin{tabular}{|c|c|c|}
\hline No. & Variable name & The meaning of variable \\
\hline 5 & imWip & The number of job in IM zone \\
\hline 6 & imLine & $\begin{array}{c}\text { The number of job waiting to be } \\
\text { processed }\end{array}$ \\
\hline 7 & imOccupation & $\begin{array}{c}\text { The sum of time of all jobs } \\
\text { to be processed }\end{array}$ \\
\hline 8 & imHot & $\begin{array}{c}\text { The number of hot job in IM } \\
\text { district }\end{array}$ \\
\hline 9 & imbottleneck & $\begin{array}{c}\text { The number of bottleneck in IM } \\
\text { district }\end{array}$ \\
\hline
\end{tabular}

B. Varible Selection and Prediction Model Construtct

Considering the dispatching rules many manufacturing factories use is FIFO, we produce 8 months simulation data at different situation under rule of FIFO to predict remaining cycle time. There are more than 60000 samples of remaining time. In order to reduce the time of modeling and 
make the model more robust, we must preprocess the data before modeling.

First of all, considering the data which is so close to each other may result in overfittings, we can reduce the data from two aspects. In the first method, this paper reduces the number by a random method by reducing about $1 / 2$ quantity of data. Another method adopts the variable selection. Here we use correlation analysis to find the variables correlated with remaining cycle time. As to the prediction model, the selection method finally chosen is random forests which needs more variables to be random selected. So this paper chooses the parameter of correlation analysis to be 0.052 . After correlation analysis, 12 variables are chosen as shown in table III, where ET2 strict is the wet etching district, ET1 is the dry etching district, DF district is the diffusion district.

TABLE III. THE VARIABLE IN IM DISTRICT

\begin{tabular}{|c|c|c|}
\hline No. & Variable name & The meaning of variable \\
\hline 2 & remainstep & $\begin{array}{c}\text { The number of steps remaining to } \\
\text { be processed }\end{array}$ \\
\hline 3 & prodid & The name of product \\
\hline 20 & dfWIP & The wafer of job in DF district \\
\hline 21 & dfLine & $\begin{array}{c}\text { The number of job waiting in DF } \\
\text { district }\end{array}$ \\
\hline 22 & dfOcupation & $\begin{array}{c}\text { The sum of time of all jobs to be } \\
\text { processed in DF district }\end{array}$ \\
\hline 24 & dfBottleneck & $\begin{array}{c}\text { The number of bottleneck in DF } \\
\text { district }\end{array}$ \\
\hline 25 & Et1Wip & $\begin{array}{c}\text { The wafer of job in ET1 district } \\
\text { district }\end{array}$ \\
\hline 26 & Et1Line & $\begin{array}{c}\text { The number of job waiting in ET1 } \\
\text { The sum of time of all jobs to be } \\
\text { processed in ET1 district }\end{array}$ \\
\hline 27 & Et1Ocupation & $\begin{array}{c}\text { The wafer of job in ET2 district } \\
\text { district }\end{array}$ \\
\hline 30 & Et2Wip & $\begin{array}{c}\text { The number of job waiting in ET2 } \\
\text { The sum of time of all jobs to be } \\
\text { processed in ET2 district }\end{array}$ \\
\hline 31 & Et2Line & \multicolumn{2}{|c|}{} \\
\hline 32 & Et2Ocupation &
\end{tabular}

Then, this paper uses the reduced data to model the remaining cycle time on MATLAB. In order to make the prediction fast, we choose the random forests model to establish the remaining cycle time model. Through establishing more than one model and then incorporate the results of each model, the disadvantage of overfittings caused by single model is overcome. The process of establishing random forest is much like bagging. If we know some ensemble methods, we can understand it easily.

1) The process of modeling is shown as follow:.

a) Select $N$ samples at random.

b) At every split point of a tree, select $M$ features radomly, choose the best feature to classify the samples. This process will be stopped when the smples at certain node of the tree is same or the number of samples at certain node reaches the minmize amout.

c) Repeat the above two steps construct some trees

d) Incorporate the result of everty tree.
From the process of establishing the ensemble model, we can find that the number of trees and the number of minimize samples at certain node determine the depth of tree and the time of modeling or prediction. Figure2 shows how these two factors influence the accuracy. From this, we can see when the number of trees in the forests is 20 and the minimum leaf is about 5 , the MSE is about 5 days. This accuracy can satisfy the need of dynamic dispatching rules.

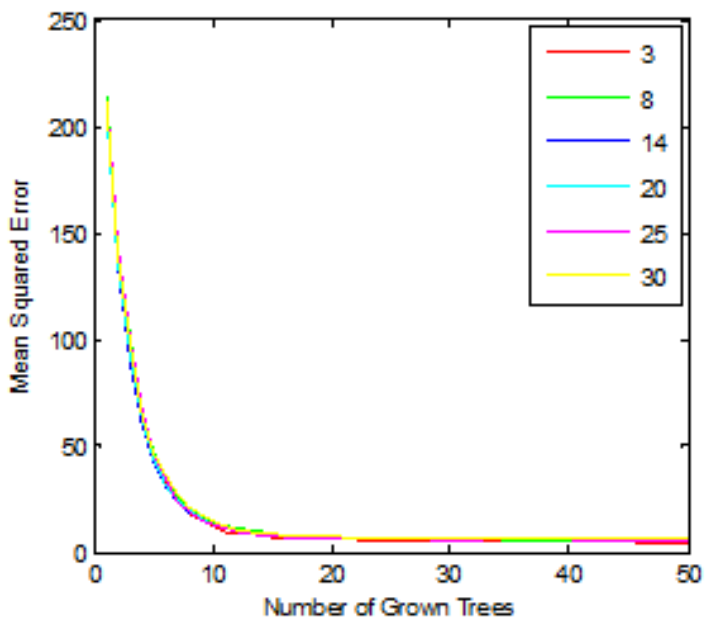

Figure 2. Different number of minimum leaf and different number of trees to the accuracy of prediction.

\section{INCORPORATE THE PREDICTION MODEL TO DYNAMIC DISPATCHING RULE}

From figure 1 , we can see if we replace $R_{i}^{n} * F_{n}$ in equation (1) the prediction of remaining cycle time, the dispatching rules will be more reasonable. In order to obtain the result of prediction value in MATLAB, we used the activex interface on simulation model to communicate with MATLAB.

After implement the communication with each other, we found it is so time consuming when simulation factory runs if we use the prediction model too fluently. So we only use the prediction model when the equipment is bottleneck to system. In our research, we define the equipment whose utilization is bigger than 0.7 as bottleneck equipment.

Finally, the dispatching rule with remaining cycle time prediction is established.

\section{RESULT}

In our research, we not only study the result between dynamic dispatching rule without cycle time prediction (DYMIC) and the rule incorporate the remaining cycle time to the rule(DYMICCT), we also ran the simulation with several common dispatching rules. The simulation time is 120 days, the performance of ODR is the average ODR of all products, the performance of MOV is the average MOV, also the throughput is the average value, so it is not an integer.

1) Rules and Therir Instituion as Follows:

a) EDD rules: the job with minimize dead line should be first processed. 
b) FIFO rules: the job which reached the buffer in advance should be first processed.

c) SPT rules: the job whose process time is shortest shouble be first processed.

d) SRPT rules: the job whose remaing process time is shortest should be first processed.

e) LPT rules: the job whose process time is longgest should be first processed.

f) LS rules: the job whose process time is shortest should be first processed.

Fig. 3 shows the performance of average MOV under different WIP and different dispatching rules. Fig. 4 shows the performance ODR with different WIP and different dispatching rules. Fig. 5 shows the throughput of the semiconductor manufacturing system under different rules. Fig. 6 shows the variance of the cycle time when WIP is 6500 .

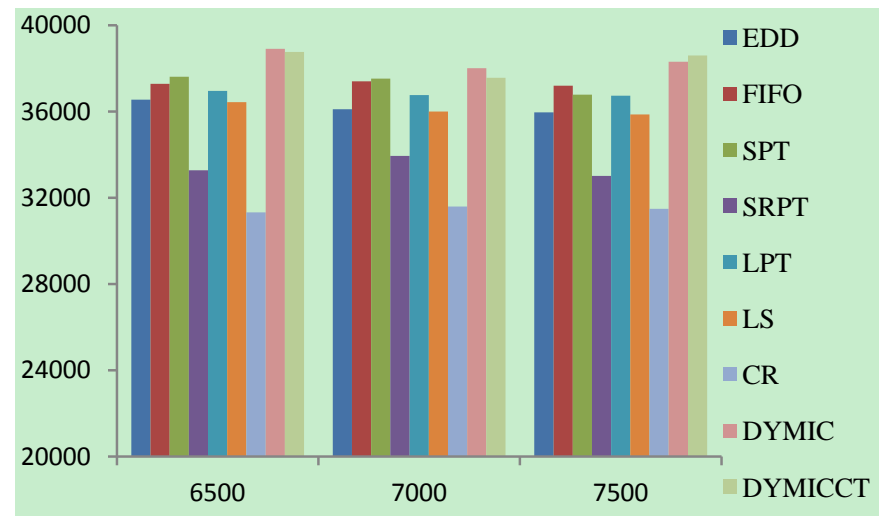

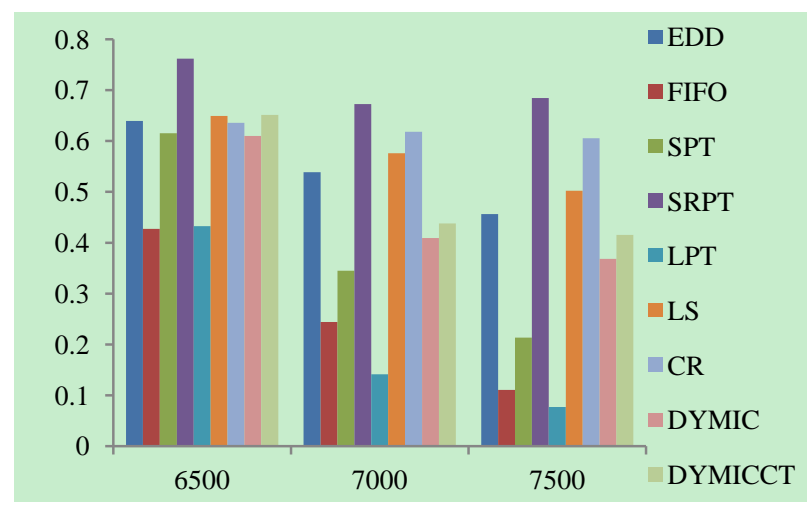

Figure 4. The performance of ODR.

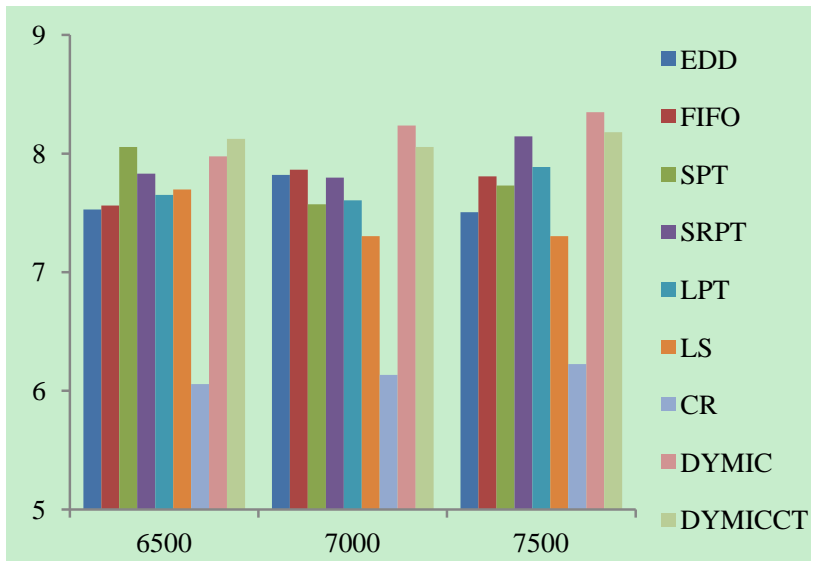

Figure 5. the performance of average throughput.

Figure 3. The performance of MOV.

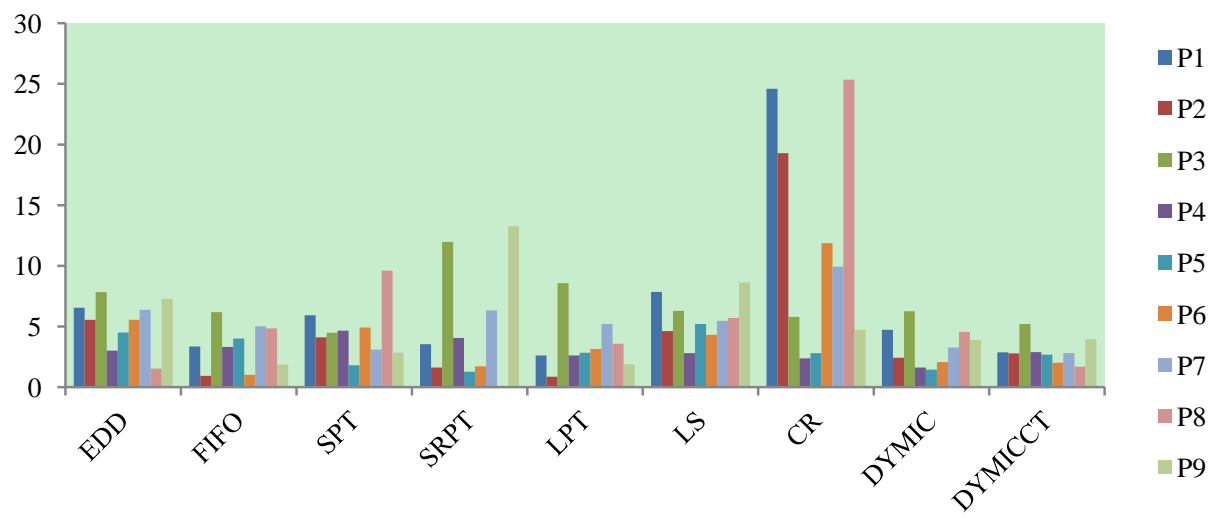

Figure 6. The variance of cycle time of different product.

In these tables we can see although the rule SRPT has advantage over other rules on the whole, but when we see the simulation result, the product with longest cycle time was never produced, that means the good performance of other index was get by sacrificing some products, so that unreasonable in real factory.

We can see there is relationship among three performance index, which is when we use some rules to improve the MOV, the other two may decrease. 
We can also see the DYMIC rule has advantage in both throughput and MOV, but in ODR, the performance is very poor comparing with other rules. But when we incorporate the remaining cycle time prediction into the DYMIC, the rule called DYMICCT, the performance of ODR was improved by $8 \%$ at average, and the performance of MOV decreased a little, but it is worthwhile. The ODR decreased when WIP at level 7500 and 7000, which is over load in the real factory, so these scenes seldom occurred.

At last, we can see the variance of cycle time from Table VII, in this table the column names P1 to P9 respect nine different products. The variance of cycle time of our novel method is range from 1.69 to 5.2 which is quite small comparing with other methods. That is meaning the product line will be more stable if we incorporate the cycle time prediction to dispatching rules.

\section{CONCLUSION}

In this paper, we study the remaining cycle time prediction in multi-product environment, and incorporate it with the dispatching rules.

We ran simulation under the rule of FIFO, for the dispatching rule in real semiconductor production line is FIFO. By simulating 8 mouths, we got enough data to model the prediction model. After doing the experiments, we can draw following conclusions:

1) About modeling: The prediction accuracy of random forests depend on the number of variables, the number of trees in forests and the number of minimize leaf in the tree. Establishing a random forests model is just like the bagging method, the only difference is in split point the random forests will select some variables at random, therefor when ding the correlation analysis, we remain enough variables for the model to select. After much MATLAB simulation we found the best number of trees is about 20, and the minimize left in the tree is about 5 , under such condition, the MSE of random forests is 5 days.

2) About perpformance: We found the dynamic rules is good at improving the performance of MOV and throughput, but in ODR it is very poor, so we study the influence caused by incorporating the remaining cycle time prediction model to the rules. Considering if we use the prediction frequently, the program will be time-consuming. We only use the prediction model in bottleneck equipment. After much simulation, we compared with other different rules under different level of WIP. We found the rules of DYMICCT is better than DYMIC at the performance index of ODR, comparing with other rules, this rule can improve the index of MOV and throughout. At last, when we compare the variance of cycle time we can see most product has a small variance under our novel rules, so we can get the conclusion that our rule can not only improve the performance index of product line but also can improve the stability of product line.

\section{ACKNOWLEDGMENT}

I would like to express my gratitude to all those who helped me during the writing of this article. And the article is supported by National Natural Science Foundation of China (No. 51475334)

\section{REFERENCES}

[1] ChiaYu Chang, Ku Hao Chang, "An Integrated and Improved Dispatching Approach to Reduce Cycle Time of Wet Etch and Furnace Operations in Semiconductor Fabrication,” IEEE Control System Magazine, pp. 33-40, Dec 1994.

[2] Jijie Deng, Zhengcai Cao, and Min Liu, “A bottleneck prediction and rolling horizon scheme combined dynamic scheduling algorithm for semiconductor wafer fabrication,” Computer Integrated Manufacturing Systems, pp. 33-35, 1997.

[3] Li Shi, Xiaohui Zhang,Li Li., "Simulation and analysis of scheduling rules for semiconductor manufacturing line," IEEE International Conference on Industrial Technology,pp. 1-5, 2008.

[4] PeiChann Chang, Yen-Wen Wang andChen-Hao Liu, "Combining SOM and GA-CBR for Flow Time Prediction in Semiconductor Manufacturing Factory,”Germany: Trans Tech Publications, pp. 123126, 2011.

[5] Hsin-Chieh Wu and Toly Chen,” A Fuzzy-Neural Ensemble and Geometric Rule Fusion Approach for Scheduling a Wafer Fabrication Factory," Mathematical Problems in Engineering, pp.1-15, 2013

[6] Yair Meidan, Boaz Lerner and Gad Rabinowitz, "Cycle-Time Key Factor Identification and Prediction in Semiconductor Manufacturing Using Machine Learning and Data Mining.,” International Journal of Production Research,pp. 1621-1638, 2004. 